\title{
Numerical study of rainbows and glories in water-drop clouds
}

\author{
S. M. PRIGARIN*, K. B. BAZAROV ${ }^{\dagger}$, M. KERSCHER ${ }^{\dagger}$ \\ and U. G. OPPEL ${ }^{\dagger}$
}

\begin{abstract}
In this paper we study scattering phase functions in water-drop clouds for various distributions of droplet size and various conditions of glory, rainbow and corona formation, and discuss the hypothesis proposed by A. N. Nevzorov that a considerable amount of water in cold clouds can exist in a specific phase state with the refractive index $\approx 1.8$ (so called A-water). Polarization and angular distributions are studied by the Monte Carlo method for radiation reflected by cloud layers with drops of water or hypothetical A-water taking into account multiple scattering. Computational results make it possible to develop procedures for analysis of microphysical structure of clouds and confirmation or disproof of the existence of A-water.
\end{abstract}

Such phenomena as rainbows, glories, or coronas appear due to peculiarities of light scattering on water droplets. The dependence of the distribution density of the intensity of scattered radiation on the angle between incident and scattered photons is called a scattering phase function. The scattering phase function of water-drop clouds is determined by the wavelength and size distribution of droplets. If all cloud drops were of the same size, then the whole cloudy sky would be filled with colored rings of various intensity, i.e., figuratively speaking, we would see a variety of rainbows, glories, and coronas (see Fig. 1).

In reality, the sizes of drops in clouds vary, and the corresponding phase functions are essentially smoothed. However, scattering phase functions for

\footnotetext{
*Institute of Computational Mathematics and Mathematical Geophysics, Siberian Branch of the Russian Academy of Sciences, Novosibirsk 630090, Russia. E-mail: sergeim.prigarin@gmail.com

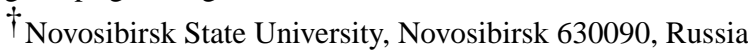

+Ludwig-Maximilian University of Munich, Munich 80539, Germany. E-mail: kersher@mathematik.uni-muenchen.de,oppel@mathematik.uni-muenchen.de

The work was supported by the Russian Foundation for Basic Research (12-05-00169).
} 

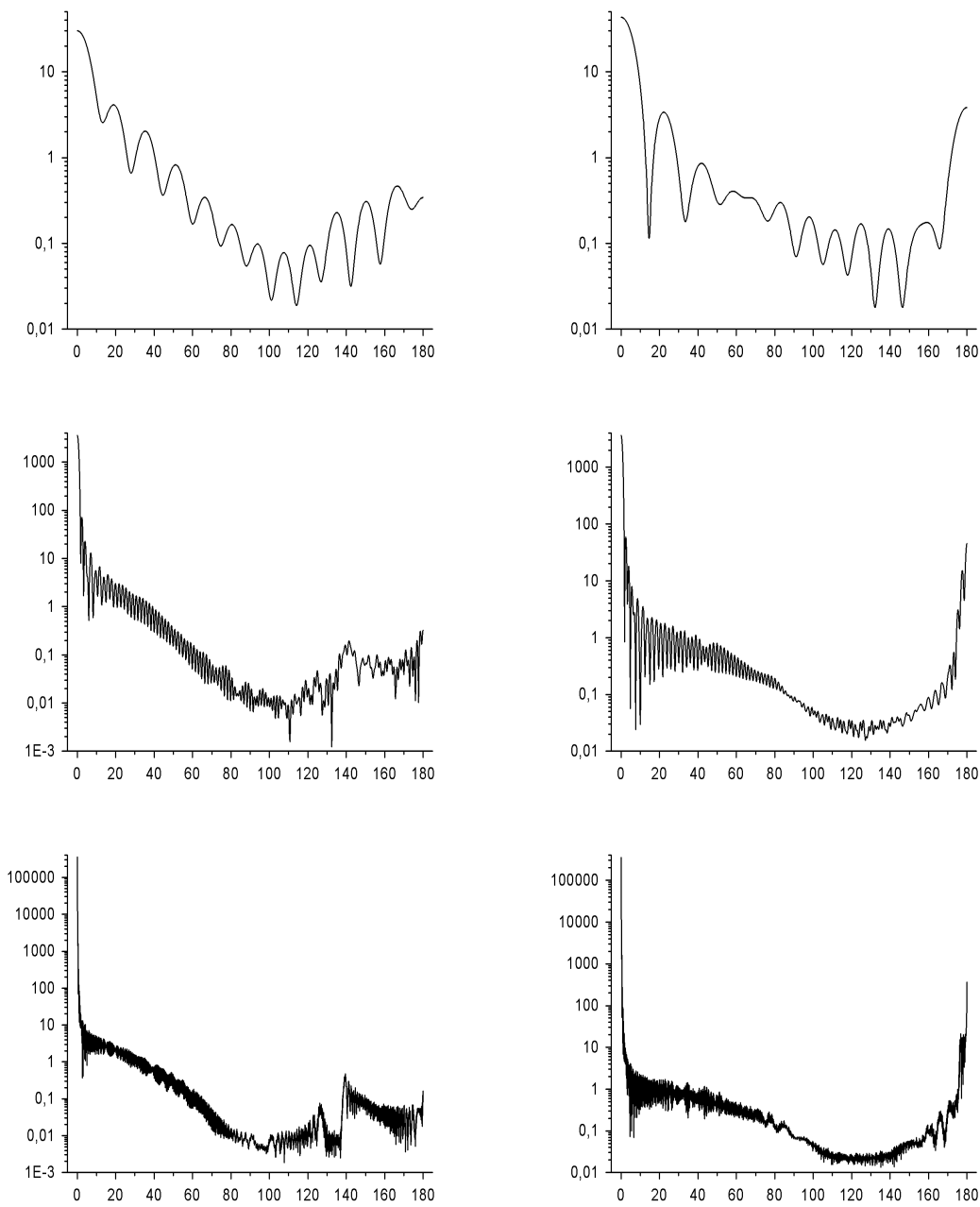

Figure 1. Scattering phase functions with wavelength $0.53 \mu \mathrm{m}$ for a monodisperse medium containing drops of water (left) and A-water (right) with the radius $1 \mu \mathrm{m}$ (upper row), $10 \mu \mathrm{m}$ (middle row) and $100 \mu \mathrm{m}$ (lower row).

actual water-drop aerosols can also contain oscillations observed ascoronas, rainbows, and glories (see the examples of phase functions presented below). Recall that coronas can be observed around the light sources (the Sun, the Moon, earth sources) when the light is transmitted through semitransparent clouds and fog. In contrast to a halo caused by light scattering on ice 
crystals and having a large angular radius, coronas are explained by diffraction scattering on water droplets, and their angular radius does not usually exceed $5^{\circ}$. A glory is actually a small rainbow with the angular radius up to $20^{\circ}$, which can be observed in the mountains or in flying above clouds. However, in contrast to a rainbow, whose approximate description can be given within geometrical optics, the calculation of a glory requires the more accurate apparatus of the Mie theory.

A. N. Nevzorov [20-27] put forward a conjecture that in cold clouds a considerable part of water is in a particular amorphous state with the refractive index $\approx 1.8$ and density $\approx 2.1 \mathrm{~g} / \mathrm{cm}^{3}$ (A-water). Moreover, according to Nevzorov's opinion, such optical phenomenon as a glory can appear only in clouds containing A-water. Nevzorov's hypothesis on the existence of Awater in clouds and on the nature of glories has not got wide approval so far and was subjected to criticism by some specialists (see $[16,34])$. In particular, it was indicated in [16] that the presence of A-water in clouds is not needed for appearance of glories (see also [13, 14, 17]).

Unfortunately, Nevzorov does not offer any information concerning the size distributions of droplets used in his calculations, which makes interpretation of his results difficult. In this paper we present some calculation results for scattering phase functions under different distributions of water and A-water drop sizes, study the peculiarities of polarization of scattered radiation, the conditions causing rainbows, glories, and coronas in clouds with water and A-water, and also the influence of multiple scattering. In particular, here we present some results supplementing our previous studies [31].

In our calculations we have used the software developed at the LudwigMaximilian University of Munich (LMU) and the Institute of Computational Mathematics and Mathematical Geophysics, Siberian Branch of the RAS, Novosibirsk (ICMMG SB RAS). The computational procedures related to the Mie theory are based on the code developed by W. Wiscombe [39]. The first versions of the software were developed at the LMU, and later this software was modified at the ICMMG SB RAS for more accurate calculations and operations with arbitrary size distributions of scattering spheres. The radiation transfer subject to multiple scattering was simulated using the software developed at the ICMMG SB RAS. 


\section{Comparison of scattering phase functions for water-drop media containing water or A-water}

In this section we compare particular features of scattering phase functions for water-drop media containing water or hypothetical A-water. We have performed calculations for a series of Gaussian distributions and for some commonly accepted models of clouds and fogs.

\subsection{Scattering phase functions for normal distributions of drops sizes}

As a first example, we consider scattering phase functions in a water-drop aerosol for water and A-water with a normal distribution of the drop radii with the mean value $m=20 \mu \mathrm{m}$ and the standard deviation $\sigma=4 \mu \mathrm{m}$ (see Fig. 2). The refractive indices of water for the calculations with different wavelengths were taken from [35]. For hypothetical A-water, the dependence of the refractive index on the wavelength is not known, therefore, in our calculations we used the value 1.82 (regardless of the wavelength) proposed in Nevzorov's papers.

The main differences in scattering between the drops of water and Awater are the following.

(1) The intensity of scattering in the backward direction is greater by a few orders in the case of A-water. Scattering by water drops is more intensive (compared to A-water) near the primary rainbow (the scattering angle is about $140^{\circ}$ ) and in the forward direction up to $40^{\circ}$.

(2) The scattering phase function for drops of A-water contains local maxima only in the neighbourhood of the glory (about $180^{\circ}$ ). Looking ahead, note that a rainbow practically never appears under scattering on drops of A-water. An exception may be found in distributions with the drop size less than a micron. Below we consider the rainbow effects appearing in this case.

As was shown by the computations, all scattering phase functions for drops of water with a Gaussian distribution for the mean radius greater than $5 \mu \mathrm{m}$ have typical maxima corresponding to the primary (the scattering angle about $140^{\circ}$ ) and secondary (the scattering angle about $125^{\circ}$ ) rainbows (see Table 3 in [31]). In this case, the effect of a supernumerary rainbow is observed for more narrow distributions and larger drops. The minimum between the primary and secondary rainbows corresponding to Alexander's dark band is observed more clearly for large drops (see Fig. 3). These fig- 


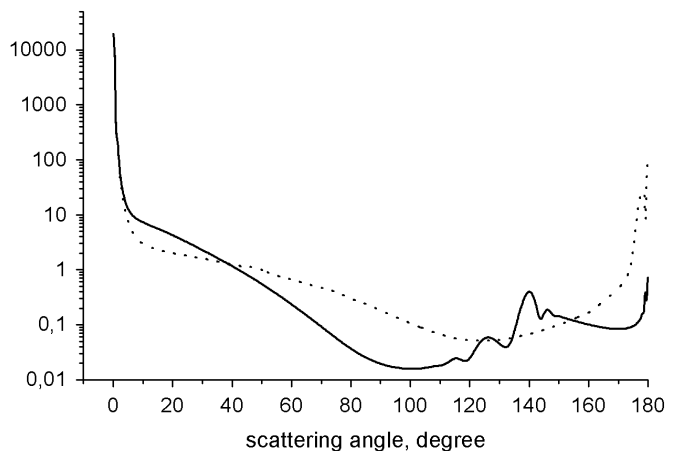

Figure 2. Scattering phase functions of light with wavelength $0.7 \mu \mathrm{m}$ for aerosol containing drops of water (solid line) and A-water (dotted line) with normal distribution of drops radii with the mean value $m=20 \mu \mathrm{m}$ and standard deviation $\sigma=4 \mu \mathrm{m}$. The local maxima correspond to a glory (about $180^{\circ}$ ), the primary rainbow (near $140^{\circ}$ ) and the secondary rainbow (near $125^{\circ}$.

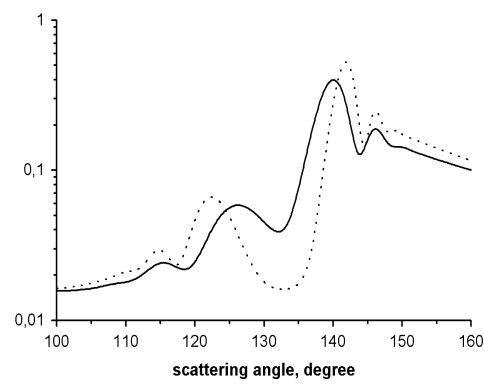

(a)

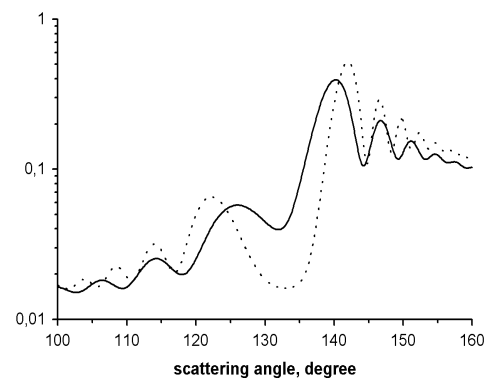

(b)

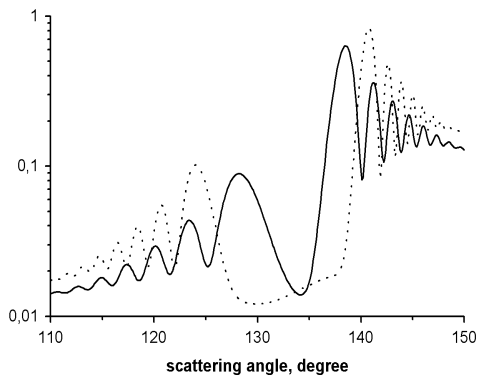

(c)

Figure 3. Scattering phase functions with wavelength $0.7 \mu \mathrm{m}$ (solid line) and $0.42 \mu \mathrm{m}$ (dotted line) in the rainbow domain for Gaussian distributions of water drops radii with the parameters $m=20 \mu \mathrm{m}, \sigma=4 \mu \mathrm{m}$ (a), $m=20 \mu \mathrm{m}, \sigma=1 \mu \mathrm{m}$ (b), $m=80 \mu \mathrm{m}, \sigma=4 \mu \mathrm{m}$ (c). It is seen that a supernumerary rainbow appears for more narrow distributions and larger drops. 
ures demonstrate a different pattern of color alternation in the primary and secondary rainbows (the wavelength of $0.7 \mu \mathrm{m}$ corresponds to the red color, 0.42 corresponds to the violet color).

Scattering phase functions in the domain of the glory are essentially different for the cases of water and A-water drops (see Figs. 4 and 5). For example, for the normal distribution of water drop radii with the parameters $m=20 \mu \mathrm{m}$ and $\sigma=4 \mu \mathrm{m}$ a glory is formed with the angular radius approximately $1^{\circ}$ and the same color alternations as in the primary rainbow (see Fig. 4a). Additional wider rainbow rings appear for more narrow distributions, however, the colors of those rings alternate not in the same manner as in the brightest inner ring (see Figs. 4b, d, f). If the mean radius of the drops is halved, the angular radius of the main inner ring of the glory increases approximately twice [31]. The same regularity is observed for glories on drops of A-water. However, in comparison with ordinary water, glories in A-water have a distinctly greater angular radius for the same distributions of drop sizes. For moderate radii of aerosol drops equal to 5 , $10,20 \mu \mathrm{m}$ the multiplicity of glories for A-water is generally less than for water. (The multiplicity of glories means the number of the local minima of the phase function near $180^{\circ}$.) If the mean radius of drops is greater, the multiplicity of glories for A-water is greater than for water. In addition, one can observe the following phenomenon: for sufficiently wide distributions, the inner rings become smoother and disappear, and only one pronounced outward ring is left (see Fig. 5e). This phenomenon is not observed for scattering on water drops. In total, the brightness of a glory for A-water drops is greater by an order of magnitude than for water drops.

For coronas we can indicate the following regularities (see Fig. 6): (1) if the mean size of drops decreases (with a constant ratio of the mean and standard deviation), the angular radius of the coronas increases; (2) if the variance of distribution decreases, the color contrast and the angular radius increase (due to the appearance of additional colored rings). In this case the forms of scattering phase functions for water and A-water drops practically coincide for the same distributions (see Fig. 6d).

In theory, interesting optical phenomena may appear in water-drop aerosols with drop sizes less that $2 \mu \mathrm{m}$ (see Fig. 7). In particular, some phenomena similar to rainbows of the third and fourth orders are quite probable (see information related to rainbows of the third an fourth orders in $[8,38])$. 


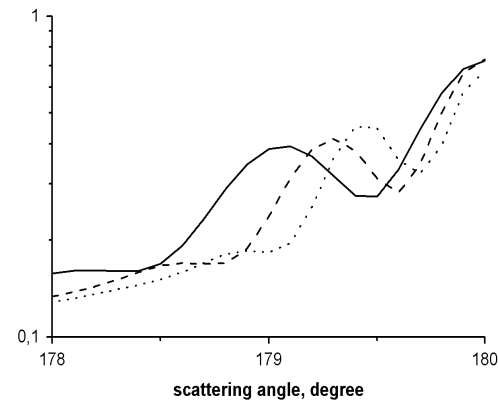

(a)

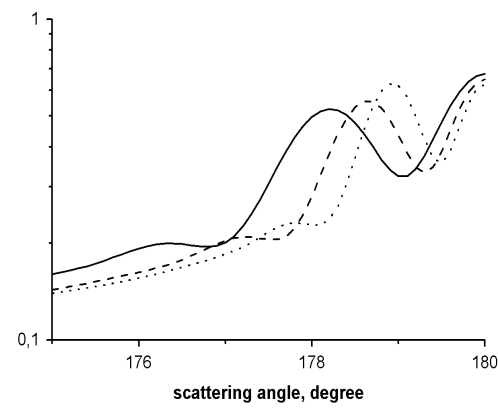

(c)

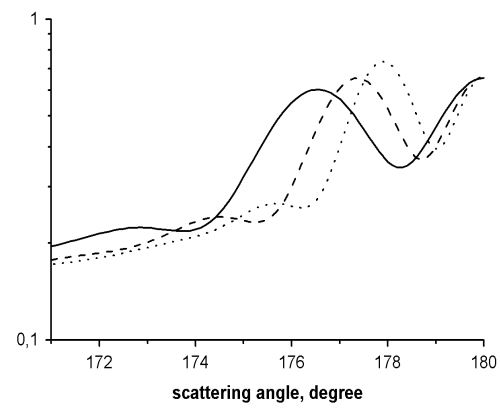

(e)

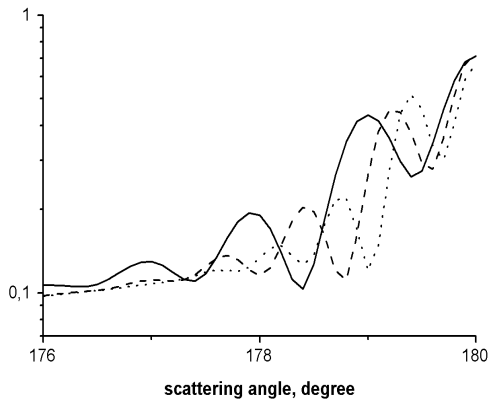

(b)

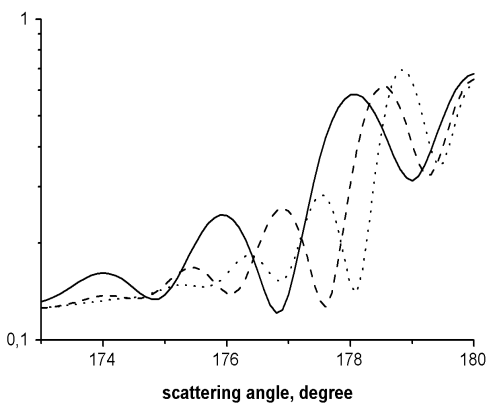

(d)

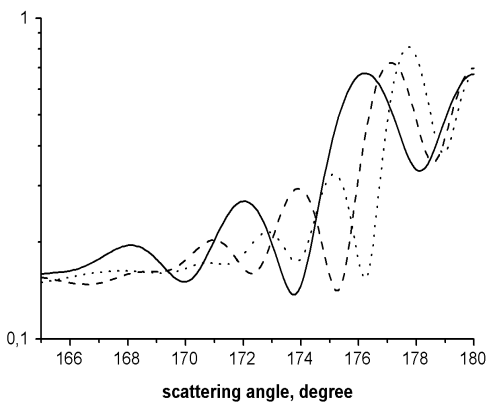

(f)

Figure 4. Scattering phase functions with the wavelength $0.7 \mu \mathrm{m}$ (solid line), $0.53 \mu \mathrm{m}$ (dashed line), and $0.42 \mu \mathrm{m}$ (dotted line) in the glory domain for Gaussian distributions of water drops radii with the parameters $m=20 \mu \mathrm{m}, \sigma=4 \mu \mathrm{m}$ (a), $m=20 \mu \mathrm{m}, \sigma=2 \mu \mathrm{m}$ (b), $m=10 \mu \mathrm{m}, \sigma=2 \mu \mathrm{m}(\mathrm{c}), m=10 \mu \mathrm{m}, \sigma=1 \mu \mathrm{m}(\mathrm{d}), m=5 \mu \mathrm{m}, \sigma=1 \mu \mathrm{m}(\mathrm{e}), m=5 \mu \mathrm{m}$, $\sigma=0.5 \mu \mathrm{m}(\mathrm{f})$. 


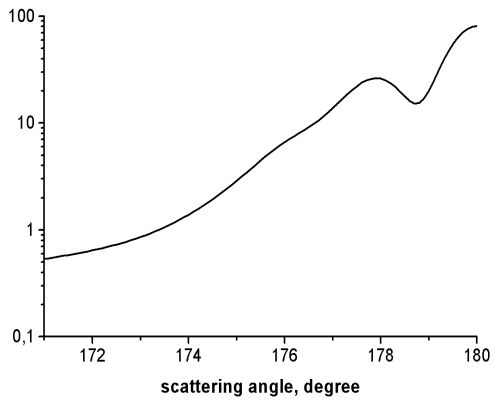

(a)

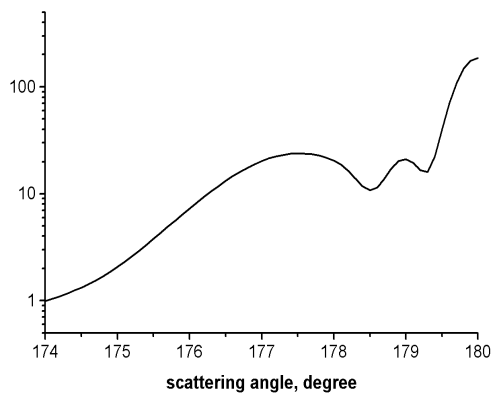

(c)

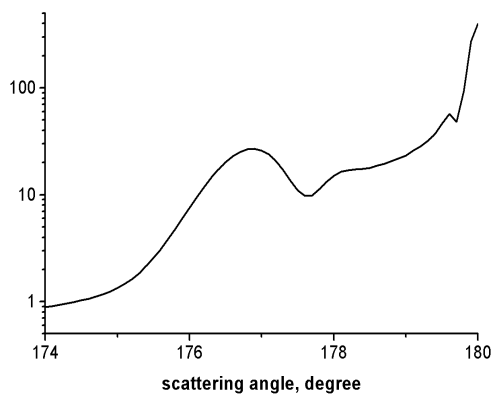

(e)

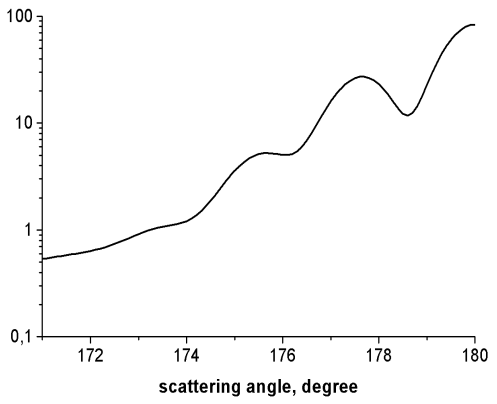

(b)

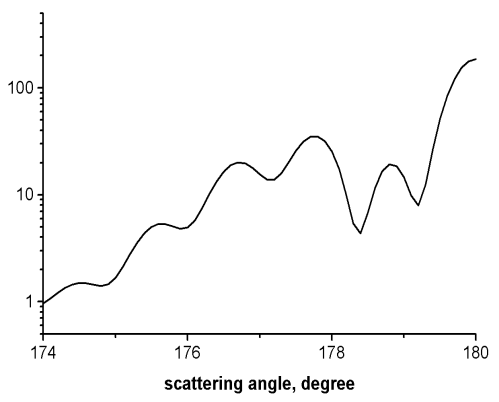

(d)

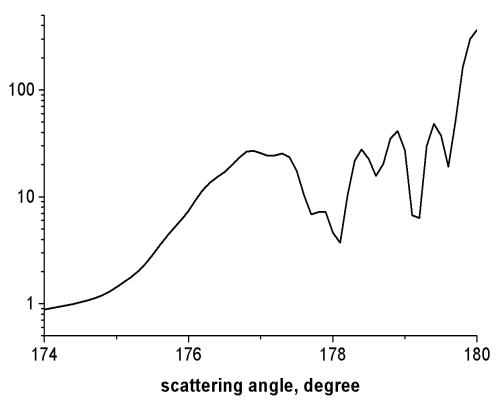

(f).

Figure 5. Scattering phase functions with the wavelength $0.53 \mu \mathrm{m}$ in the glory domain for Gaussian distributions of A-water drops radii with the parameters $m=10 \mu \mathrm{m}, \sigma=2 \mu \mathrm{m}$ (a), $m=10 \mu \mathrm{m}, \sigma=1 \mu \mathrm{m}(\mathrm{b}), m=20 \mu \mathrm{m}, \sigma=4 \mu \mathrm{m}(\mathrm{c}), m=20 \mu \mathrm{m}, \sigma=1 \mu \mathrm{m}(\mathrm{d}), m=40 \mu \mathrm{m}$, $\sigma=16 \mu \mathrm{m}(\mathrm{e}), m=40 \mu \mathrm{m}, \sigma=2 \mu \mathrm{m}$ (f). 


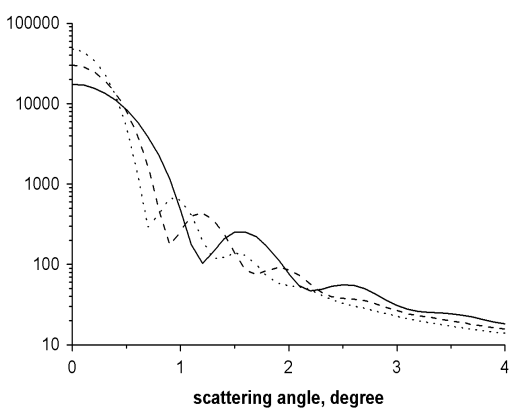

(a)

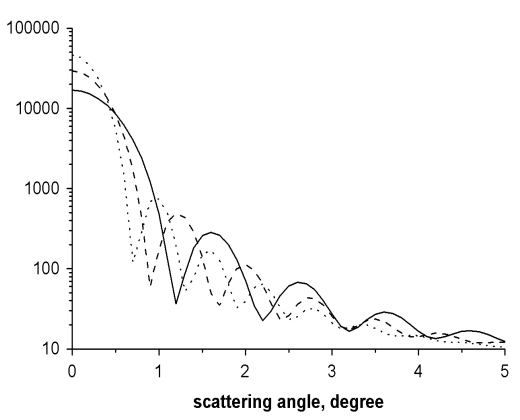

(c)

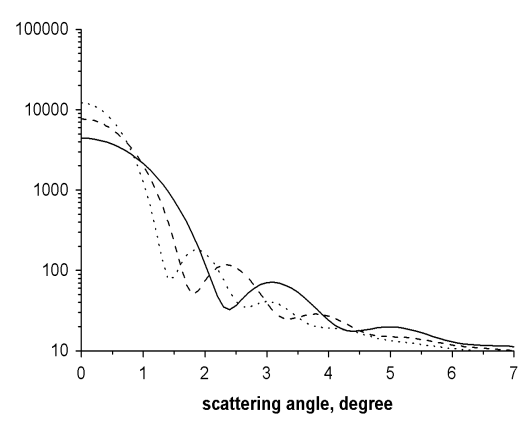

(b)

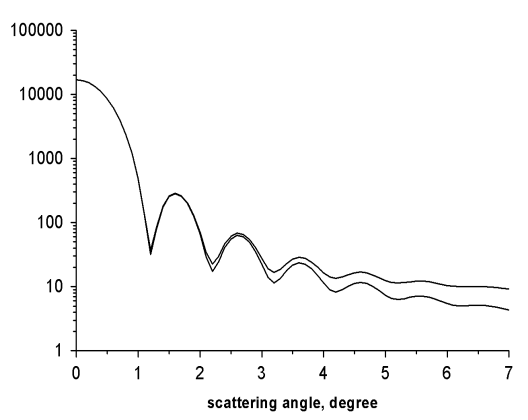

(d)

Figure 6. Scattering phase functions with wavelength $0.7 \mu \mathrm{m}$ (solid line), $0.53 \mu \mathrm{m}$ (dashed line), and $0.42 \mu \mathrm{m}$ (dotted line) in the corona domain for Gaussian distributions of water drops radii with the parameters $m=20 \mu \mathrm{m}, \sigma=2 \mu \mathrm{m}$ (a), $m=10 \mu \mathrm{m}, \sigma=1 \mu \mathrm{m}$ (b), $m=$ $20 \mu \mathrm{m}, \sigma=1 \mu \mathrm{m}$ (c) and (d). Figure (d) presents the phase function for water drops (upper line) together with the corresponding phase function for A-water (lower line).

\subsection{Scattering phase functions for cloud and fog models}

Comparative calculations of phase functions for aerosols with water and A-water drops have been performed for a series of distributions used in description of various models of clouds and fogs. We considered six OPAC models [9], four MODTRAN models [1,3], and 10 models from [6, 7, 36]. For all models we used an approximation of drop sizes by a modified Gamma-distribution with the density

$$
w(r)=A r^{\alpha} \exp \left(-B r^{\gamma}\right), \quad B=\frac{\alpha}{\gamma r_{\text {mod }}^{\gamma}} .
$$

Here $A, \alpha, B, \gamma$ are the parameters of the distribution density of the 


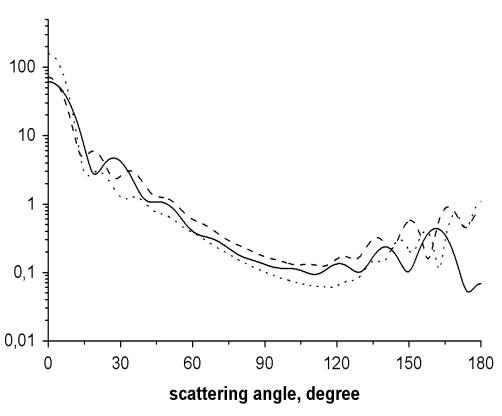

(a)

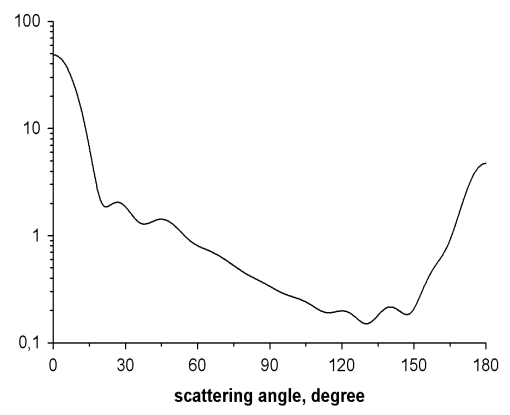

(b)

Figure 7. Scattering phase functions with wavelength $0.7 \mu \mathrm{m}$ (solid line), $0.53 \mu \mathrm{m}$ (dashed line), and $0.42 \mu \mathrm{m}$ (dotted line) for Gaussian distributions of drops radii for water (a) and A-water (b) with the parameters $m=1 \mu \mathrm{m}, \sigma=0.07 \mu \mathrm{m}$.

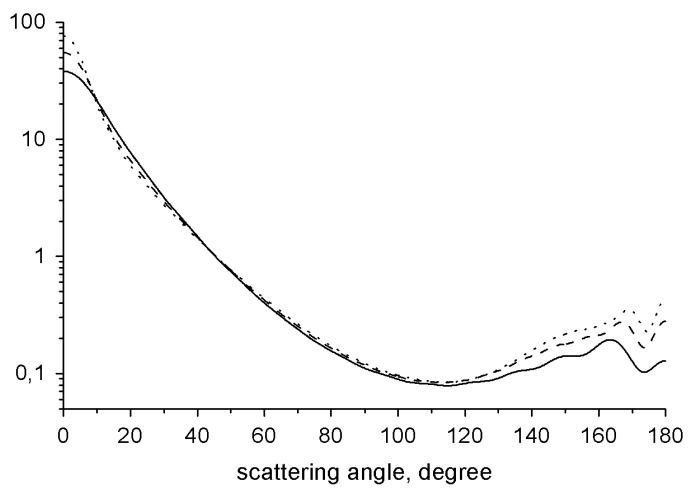

Figure 8. Scattering phase functions of light with wavelength $0.7 \mu \mathrm{m}$ (solid line), $0.53 \mu \mathrm{m}$ (dashed line), and $0.42 \mu \mathrm{m}$ (dotted line) for 'Haze.M' model.

concentration $w(r)$ of drops in $\mathrm{cm}^{3}$ with respect to the radius $r$ measured in $\mu \mathrm{m} ; r_{\text {mod }}$ is the modal radius of the drops. The results of calculations were briefly presented in [31]. Note the following issues most essential from our viewpoint.

1. Practically all phase functions for popular cloud models (with water drops) have local maxima in the domains of the rainbow and the glory and resemble the solid line shown in Fig. 2. For some models, a white rainbow is typical (maxima for different wavelengths practically coincide). In particular, a white rainbow is 'observed' for models C3 from [6] and for the model of moderate radiation fog [36]. The models 'Haze.H', 'Haze.L', and 
'Haze.M', stand apart. These models differ from the others by very small drops sizes (about a micron) and their phase functions do not have local maxima in the domain of the rainbow. In this case, glories of an abnormally large radius are observed for the models 'Haze.L' and 'Haze.M' (see Fig. 8).

2. For the same distributions of drop radii, the scattering phase functions have an absolutely different form if we suppose that the aerosol contains A-water with the refraction index 1.82, see the phase function indicated by the dashed line in Fig. 2. In this case, most of the phase functions do not have local maxima (neither in the domain of rainbow, nor in the glory domain) and only for several models one can observe local maxima in the glory domain. The most expressed glory is the one for the cloud model 'Cumulus OPAC Maritime' with the parameters $\alpha=4, B=0.00713, \gamma=$ $2.34, r_{\mathrm{mod}}=10.399 \mu \mathrm{m}$ in $(1.1)$.

\section{Peculiarities of radiation polarization under single scattering}

Radiation scattering subject to polarization is described by a Mueller matrix. Suppose a photon described by the Stokes vector $S^{\prime}=\left(I^{\prime}, Q^{\prime}, U^{\prime}, V^{\prime}\right)$ with the motion direction $\omega^{\prime}$ is scattered at some spatial point $r^{\prime}$. Then the Stokes vector $S=(I, Q, U, V)$ for the radiation intensity at the point $r=r^{\prime}+R \omega$ scattered from the point $r^{\prime}$ in the direction $\omega$ is described at the distance $R=\left\|r-r^{\prime}\right\|$ from the scattering point by the expression

$$
S(\omega)=M\left(\omega^{\prime}, \omega, r^{\prime}\right) S^{\prime} \frac{1}{R^{2}}
$$

where $M$ is the Mueller matrix. Recall (see, e.g., [30]) that the unique determination of the second and third components of the Stokes vector requires a specification of the additional reference vector orthogonal to the motion of the photon. It is assumed in (2.1) that the Stokes vectors $S^{\prime}$ and $S$ have a common reference vector orthogonal to the scattering plane containing the vectors $\omega^{\prime}$ and $\omega$. The Mueller matrix has the following form for water-drop clouds with spherical drops $[4,10]$ :

$$
M\left(\omega^{\prime}, \omega\right)=\left[\begin{array}{cccc}
M_{11} & M_{12} & 0 & 0 \\
M_{12} & M_{11} & 0 & 0 \\
0 & 0 & M_{33} & M_{34} \\
0 & 0 & -M_{34} & M_{33}
\end{array}\right], \quad\left\langle\omega^{\prime}, \omega\right\rangle \in(-1,1)
$$




$$
\begin{aligned}
M\left(\omega^{\prime}, \omega\right) & =\left[\begin{array}{cccc}
M_{11} & 0 & 0 & 0 \\
0 & M_{11} & 0 & 0 \\
0 & 0 & M_{11} & 0 \\
0 & 0 & 0 & M_{11}
\end{array}\right], \quad\left\langle\omega^{\prime}, \omega\right\rangle=1 \\
M\left(\omega^{\prime}, \omega\right) & =\left[\begin{array}{cccc}
M_{11} & 0 & 0 & 0 \\
0 & M_{11} & 0 & 0 \\
0 & 0 & -M_{11} & 0 \\
0 & 0 & 0 & -M_{11}
\end{array}\right], \quad\left\langle\omega^{\prime}, \omega\right\rangle=-1
\end{aligned}
$$

and is calculated by Mie formulas.

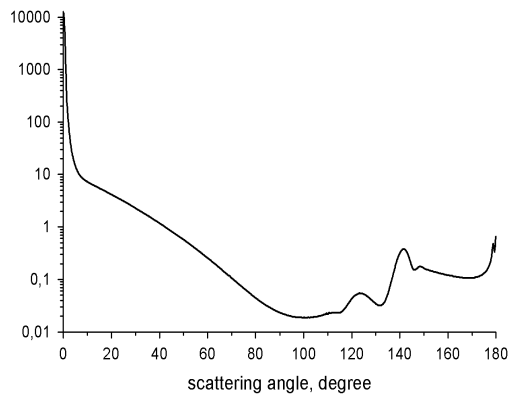

(a)

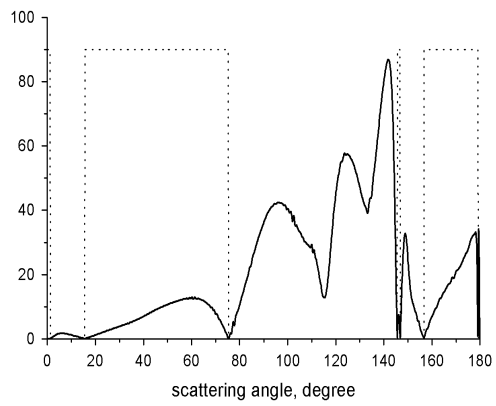

(c)

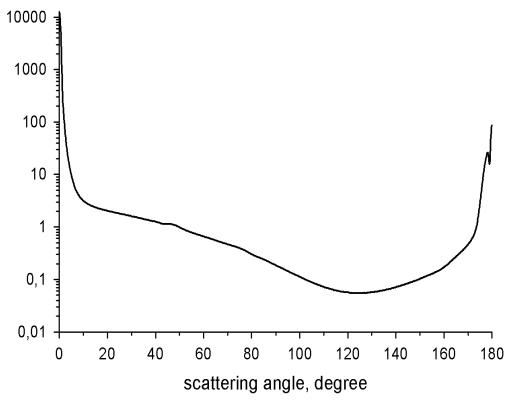

(b)

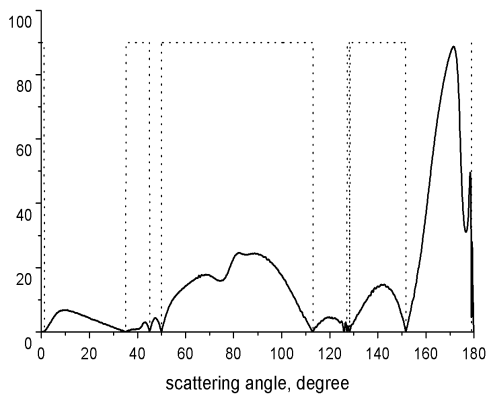

(d)

Figure 9. The figure presents the scattering phase functions with wavelength $0.53 \mu \mathrm{m}(\mathrm{a}, \mathrm{b})$ and the polarization degree in percent (c, d) for water (a, c) and A-water (b, d) according to the 'OPAC Cumulus Maritime' model. Polarization is radial under the dotted line and tangential in other regions. 
The polarization of scattered radiation on water and hypothetical A-water drops is essentially different for monodisperse, as well as for polydisperse media (see Figs. 9 and 10) presenting the degree $\sqrt{Q^{2}+U^{2}+V^{2}} / I$ and the direction of polarization for single scattering of natural light. The plane of polarization of a scattered ray may coincide with the plane of scattering (radial polarization) or be orthogonal to it (tangent polarization). The domains with radial polarization in the above figures are under the rectangles drawn with a dotted line. For single scattering on water drops in clouds, the maximal degree of polarization is observed in the rainbow domain. The degree of polarization can reach $90 \%$ here and the direction of polarization is tangential. In contrast to the case of the rainbow, polarization in the glory domain is radial and its degree is essentially lower (about 30\%).

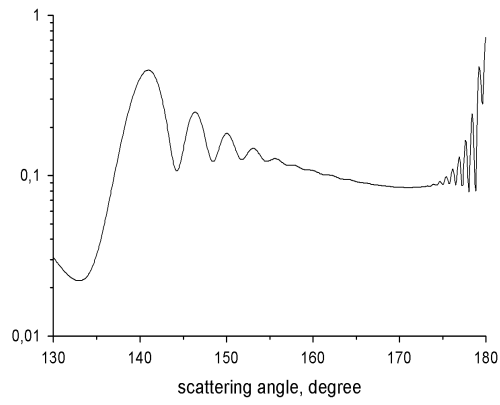

(a)

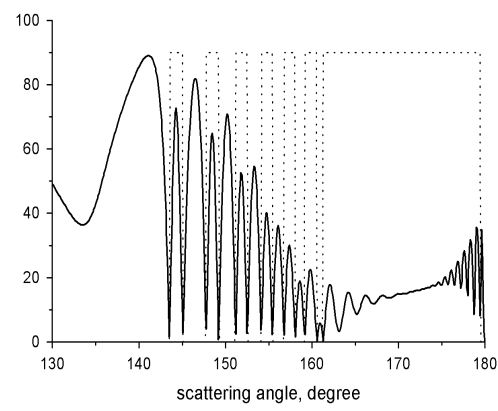

(c)

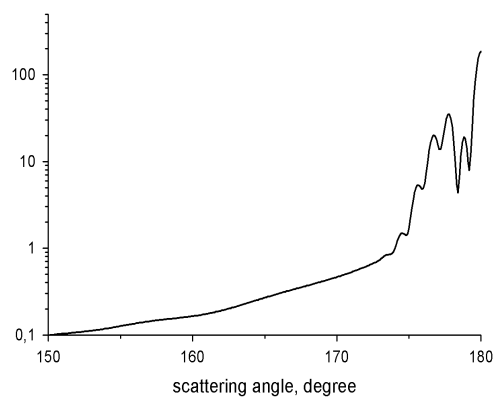

(b)

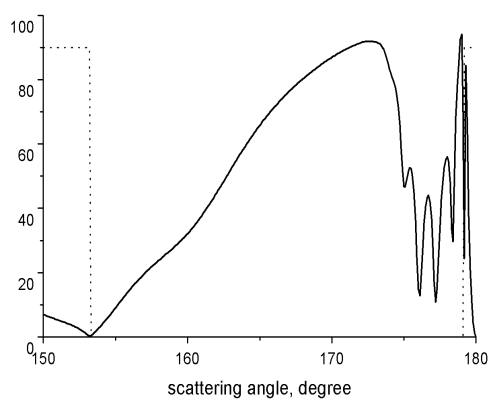

(d)

Figure 10. The figure presents the light scattering phase functions with wavelength $0.53 \mu \mathrm{m}$ $(a, b)$ and the polarization degree in percent $(c, d)$ for water $(a, c)$ and A-water (b, d) according to the Gaussian distribution of drops with the parameters $m=20 \mu \mathrm{m}, \sigma=1 \mu \mathrm{m}$. The polarization is radial under the dotted line and tangential in other regions. 
In the case of hypothetical A-water, the maximal degree of polarization (about $90 \%$ ) is observed near the scattering angles of $170^{\circ}$. In the glory domain the degree of polarization can also reach high values (for more narrow distributions of drop sizes the degree of polarization is higher). In this case the direction of polarization in these domains is tangential.

There is practically no polarization in the corona domains both for water and A-water.

Thus, it is obvious that the presence of A-water in clouds can be efficiently determined by studying the dependence of the degree and direction of polarization on the scattering angle.

\section{Influence of multiple scattering on polarization and angular distributions of radiation reflected from a cloud layer}

An interpretation of radiation field observations in a cloudy atmosphere requires taking into account multiple radiation scattering. The processes of polarized radiation transfer in scattering media are described by an equation for the Stokes vector (see, e.g., $[5,11,15,18,28,37])$. We present here the stationary polarized radiation transfer equation in its integral form with a generalized matrix kernel:

$$
\begin{aligned}
S[\rho](r, \omega)= & \int_{\Omega} \int_{R^{3}} \frac{e^{-\tau\left(r^{\prime}, r\right)}}{\left\|r-r^{\prime}\right\|^{2}} q\left(r^{\prime}\right) \sigma\left(r^{\prime}\right) L\left[\rho, \rho^{*}\right]^{-1} M\left(\omega^{\prime}, \omega, r^{\prime}\right) L\left[\rho^{\prime}, \rho^{*}\right] \\
& \times S^{\prime}\left[\rho^{\prime}\right]\left(r^{\prime}, \omega^{\prime}\right) \times \delta\left(\omega-\frac{r-r^{\prime}}{\left|r-r^{\prime}\right|}\right) \mathrm{d} r^{\prime} \mathrm{d} \omega^{\prime}+S_{0}[\rho](r, \omega) \\
\rho^{*}= & \frac{\omega^{\prime} \times \omega}{\left\|\omega^{\prime} \times \omega\right\|}, \quad r, r^{\prime} \in R^{3}, \quad \omega, \omega^{\prime} \in \Omega=\left\{\omega \in R^{3}:\|\omega\|=1\right\} .
\end{aligned}
$$

A photon (a quasimonochromatic wave) is described here by the Stokes vector $S[\rho](r, \omega)$ relative to the reference vector $\rho$, where $r$ are the photon spatial coordinates and $\omega$ is the unit direction vector of the photon motion (the reference vector $\rho$ is always orthogonal to the motion direction $\omega$ ), $S_{0}[\rho](r, \omega)$ is the Stokes vector corresponding to the volumetric distribution density of the sources. The reference vectors $\rho^{\prime}, \rho$ are determined through a certain field of reference vectors $\rho(\omega), \omega \in \Omega$, given in advance, i.e.,

$$
\rho^{\prime}=\rho\left(\omega^{\prime}\right) \perp \omega^{\prime}, \quad \rho=\rho(\omega) \perp \omega
$$


where $\delta$ is the delta-function, $q$ is the albedo of single scattering, $\sigma$ is the attenuation of the medium, $\tau\left(r^{\prime}, r\right)$ is the optical length of the interval $\left[r^{\prime}, r\right]$,

$$
\tau\left(r^{\prime}, r\right)=\int_{0}^{l} \sigma\left(r^{\prime}+s\left(r-r^{\prime}\right) / l\right) \mathrm{d} s, \quad l=\left\|r-r^{\prime}\right\| .
$$

Here $M\left(\omega^{\prime}, \omega, r^{\prime}\right)$ is the Mueller matrix, and $L\left[\rho^{\prime}, \rho^{*}\right]$ is the rotation matrix of the form

$$
L\left[\rho^{\prime}, \rho^{*}\right]=\left[\begin{array}{cccc}
1 & 0 & 0 & 0 \\
0 & \cos 2 \varphi & \sin 2 \varphi & 0 \\
0 & -\sin 2 \varphi & \cos 2 \varphi & 0 \\
0 & 0 & 0 & 1
\end{array}\right]
$$

where $\varphi$ is the angle used for rotation of the reference vector $\rho^{\prime}$ around $\omega^{\prime}$ in order to get the reference vector $\rho^{*}$. The rotation matrix describes the transformation of the Stokes vector under a change of the reference vector, i.e., $S\left[\rho^{*}\right]=L\left[\rho^{\prime}, \rho^{*}\right] S\left[\rho^{\prime}\right]$. The first element of the Mueller matrix satisfies the relation $\int_{\Omega} M_{11}\left(\omega^{\prime}, \omega, r^{\prime}\right) \mathrm{d} \omega=1$. The integrand in equation (3.1) formally reflects the following stages of radiation transfer: a collision at the point $r^{\prime}$ of a photon flying in the direction $\omega^{\prime}$, scattering in the direction $\omega$, and attenuation on the path to the point $r$.

One of the most efficient methods for solving polarized radiation transfer equations is the Monte Carlo method (see, e.g., [2, 12, 15, 19, 29, 32,33]) consisting in computer simulation of a large ensemble of random photon trajectories and statistical estimation of the required values. Here we briefly describe the Monte Carlo method for the stationary transfer equation used for our numerical experiment. For the sake of simplicity, below we assume that the optical medium is homogeneous and the albedo of single scattering is equal to one (the absorption of radiation in atmospheric clouds can be neglected for the visible range of wavelengths). The simulation of photon trajectories in the scattering medium and the recalculation of the Stokes vector are performed according to the following algorithm.

(1) Simulate the initial point $r_{0}=\left(x_{0}, y_{0}, z_{0}\right)$, the direction $\omega_{0}=\left(a_{0}, b_{0}, c_{0}\right)$, and the Stokes vector $S\left[\rho_{0}\right]\left(r_{0}, \omega_{0}\right)$ according to the source distribution and assume $n=0$.

(2) Simulate the free run length $l$ according to the distribution with the density $p_{l}(l)=\sigma \exp (-\sigma l), l>0$, where $\sigma$ is the attenuation of the scattering medium. 
(3) Assume $n=n+1$ and calculate the coordinates $x_{n}, y_{n}, z_{n}$ of the next collision of the photon with particles of the medium, i.e.,

$$
\begin{array}{cc}
x_{n}=x_{n-1}+a_{n-1} l, & y_{n}=y_{n-1}+b_{n-1} l \\
z_{n}=z_{n-1}+c_{n-1} l, & r_{n}=\left(x_{n}, y_{n}, z_{n}\right) .
\end{array}
$$

(4) The scattering of the photon at the point $r_{n}$ is simulated in the following way: the new motion direction $\omega_{n}$ of the photon is simulated according to some phase function $g\left(\omega_{n-1}, \omega_{n}\right)$ (distribution on the unit sphere of directions $\Omega), \int_{\Omega} g\left(\omega_{n-1}, \omega\right) \mathrm{d} \omega=1$, the values of the Stokes vector and the reference vector are recalculated by the formulas

$$
\begin{gathered}
S\left[\rho_{n}\right]\left(r_{n}, \omega_{n}\right)=\frac{M\left(\omega_{n-1}, \omega_{n}\right)}{g\left(\omega_{n-1}, \omega_{n}\right)} L\left[\rho_{n-1}, \rho_{n}\right] S\left[\rho_{n-1}\right]\left(r_{n-1}, \omega_{n-1}\right) \\
\rho_{n}=\frac{\omega_{n-1} \times \omega_{n}}{\left\|\omega_{n-1} \times \omega_{n}\right\|} .
\end{gathered}
$$

Here $M\left(\omega_{n-1}, \omega_{n}\right)$ is the Mueller matrix and $L\left[\rho_{n-1}, \rho_{n}\right]$ is the rotation matrix, see (3.2). Note that new reference vector (3.4) is taken orthogonal to the scattering plane containing the vectors $\omega_{n-1}, \omega_{n}$.

(5) Go to step 2. The simulation of trajectories is terminated when the photon leaves the cloud layer.

In order to simulate photon scattering, we have implemented two approaches. The first one (see, e.g., $[12,15,19,32])$ consists in the use of the first element of the Mueller matrix as the scattering phase function, i.e.,

$$
g\left(\omega_{n-1}, \omega_{n}\right)=M_{11}\left(\omega_{n-1}, \omega_{n}\right) .
$$

The second approach (see, e.g., $[2,29,33]$ ) is aimed to provide the equality

$$
I\left(r_{n}, \omega_{n}\right)=I\left(r_{n}, \omega_{n-1}\right) .
$$

Recall that we neglect the absorption, and $I$ is the first component of the Stokes vector $S$ describing the radiation intensity. In this case the phase function $g\left(\omega_{n-1}, \omega_{n}\right)$ in (3.3) used for scattering simulation is determined by the first component of the vector

$$
M\left(\omega_{n-1}, \omega_{n}\right) L\left[\rho_{n-1}, \rho_{n}\right] \frac{S\left[\rho_{n-1}\right]\left(r_{n}, \omega_{n-1}\right)}{I\left(r_{n}, \omega_{n-1}\right)} .
$$


Each of methods (3.5) and (3.7) simulating scattering has its own advantages depending on the peculiarities of the problem being solved. In our study of polarization and angular distributions of radiation reflected from a cloud layer both approaches have given practically the same results.

The main differences that are typical for single scattering on water and A-water drops are also well observed for multiple scattering. Figure 11 shows the transformation of the 'indicatrix of reflection' of radiation perpendicular to the cloud layer depending on the optical thickness of the layer. It is assumed here that the size distribution of the water drops corresponds to the 'OPAC Cumulus Maritime' cloud model.

Remark 3.1. The phase functions of single scattering $g_{s}(\theta), \theta \in[0, \pi]$, presented in Figs. 1-10 are normalized in the following way:

$$
\int_{0}^{\pi} g_{s}(\theta) \sin \theta \mathrm{d} \theta=2 .
$$

The indicatrix $g_{r}(\theta), \theta \in[\pi / 2, \pi]$, of reflection by a flat layer means the distribution of light brightness coming from a unit area of the layer illuminated by a perpendicular flow of photons (here $\theta$ is the angle between the incident ray and that reflected after multiple scattering in the layer). The phase functions shown in Fig. 11 are normalized as $\int_{\pi / 2}^{\pi} g_{r}(\theta) \sin \theta \mathrm{d} \theta=A$, where $A$ is the albedo of the cloud layer.

The results presented in Fig. 11 have been obtained by the Monte Carlo method and demonstrate smoothing of the rainbow and glory with the growth of the optical thickness of the cloud layer. Figure 12 shows how the polarization degree of radiation reflected from the cloud layer varies with the growth of the optical thickness of the layer. It is seen that for sufficiently large optical thickness the polarization degree of the reflected radiation remains noticeable both for ordinary water and for hypothetical A-water. The size distribution of water drops according to the 'OPAC Cumulus Maritime' cloud model was used here as well.

Note a typical peculiarity of angular distributions of radiation reflected by a cloud layer with A-water. The calculations for models with A-water give a pronounced distribution peak in the direction backward to the source, which is caused by a higher peak in the phase function of single scattering on drops of A-water compared to ordinary water. Although this peak is 


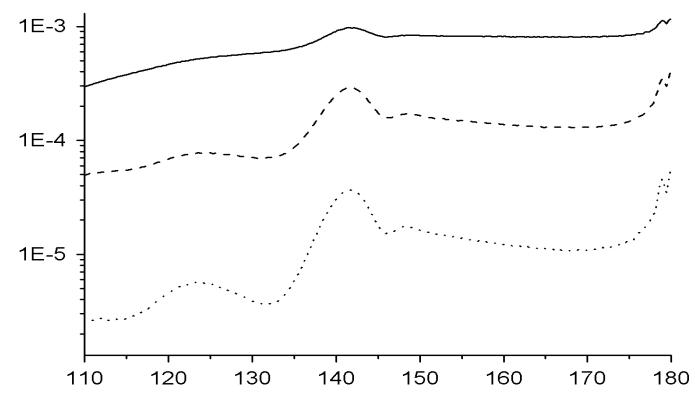

Figure 11. Light reflection indicatrices with wavelength $0.53 \mu \mathrm{m}$ for a flat cloud layer with optical density 0.1 (dotted line), 1 (dashed line), and 5 (solid line) with the distribution of water drops corresponding to the model 'OPAC Cumulus Maritime'.

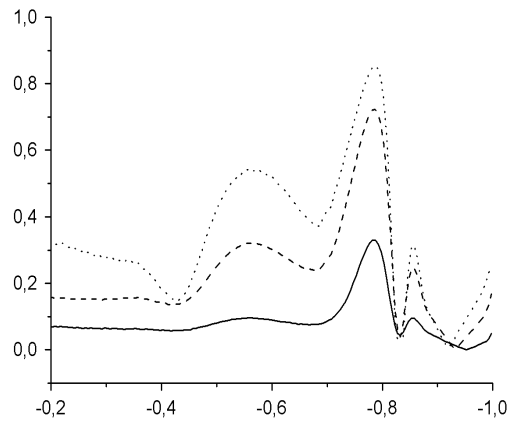

(a)

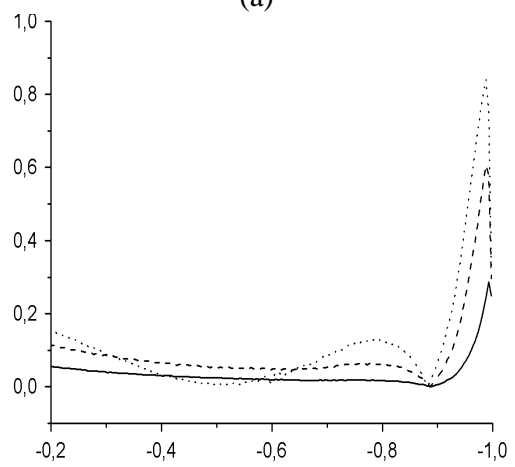

(b)

Figure 12. Polarization degree of radiation reflected by a flat cloud layer with optical thickness 0.1 (dotted line), 1 (dashed line), and 5 (solid line) with distribution of drops of water (a) and A-water (b) corresponding to the model 'OPAC Cumulus Maritime'. The dependence on the cosine of the angle between the reflected rays and the rays perpendicular to the layer is indicated. The calculations were performed by the Monte Carlo method for light with wavelength $0.53 \mu \mathrm{m}$. 
smoothed in multiple scattering, it remains rather distinguishable for large optical thicknesses and different angles of source elevation.

\section{Conclusion}

A. N. Nevzorov proposed his conjecture on the presence of A-water in cold clouds 20 years ago on the base of analysis of airplane observations. His arguments did not get support of specialists, but, at the same time, so far this conjecture has not been reliably disproved. In our opinion, this problem is rather urgent now, because in the case of discovery of water with unusual properties in the clouds we need an adaptation of the models and methods for solution of direct and inverse problems of cloudy atmosphere optics.

As was shown by our calculations (in particular, see Figs. 2, 9-11), an indirect argument in favour of Nevzorov's conjecture is that in most cases a glory on water drops must be accompanied with an intensive rainbow. However, this argument cannot be assumed as a reliable proof of the conjecture (there are known observation of a glory accompanied with a white rainbow, a rainbow can possibly be beyond the observed area, etc.). At the same time, the typical singularities of the angular distributions and polarization properties of radiation in clouds containing hypothetical A-water can be calculated using the Mie theory and statistical modelling, which gives us the ability to detect A-water reliably (if it exists) based on the brightness, degree and direction of polarization of radiation reflected from the cloud layer.

\section{Acknowledgements}

We are grateful to Anatolii Nikolaevich Nevzorov (Central Aerological Observatory, Dolgoprudny), Bernhard Mayer and Claudia Emde (LudwigMaximilians-University, Munich), Philip Laven, Tat'yana Borisovna Zhuravleva and Tat'yana Vladimirovna Bedareva (V. E. Zuev Institute of Atmospheric Optics, Tomsk) for interesting and useful discussions.

\section{References}

1. L. W. Abreu and G. P. Anderson, The MODTRAN 2/3 Report and LOWTRAN 7 Model. Prepared by Ontar Corporation for PL/GPOS, 1996.

2. S. Bartel and A. H. Hielscher, Monte Carlo simulations of the diffuse backscattering Mueller matrix for highly scattering media. Appl. Optics (2000) 39, No. 10, 1580-1588. 
3. A. Berk, L. S. Bernstein, G. P. Anderson, P. K. Acharya, D. C. Robertson, J. H. Chetwynd, and S. M. Adler-Golden, MODTRAN Cloud and Multiple Scattering Upgrades with Application to AVIRIS. Remote Sens. Environ. (1998) 65, 367-375.

4. C. F. Bohren and D. R. Huffman, Absorption and Scattering of Light by Small Particles. Wiley, New York, 1983.

5. S. Chandrasekhar, Radiative Transfer. Dover Publ., New York, 1960.

6. D. Deirmendjian, Electromagnetic Scattering on Spherical Polydispersions. American Elsevier, New York, 1969.

7. P. V. Dyachenko, Experimental Application of the Method of Mathematical Statistics to Microstructural Fog and Cloud Research. Voeikov Main Geophys. Observ., 1962 (in Russian).

8. M. Grossmann, E. Schmidt, and A. Haussmann, Photographic evidence for the thirdorder rainbow. Appl. Optics (2011) 50, No. 28, 134-141.

9. M. Hess, P. Koepke, and I. Schult, Optical properties of aerosols and clouds: the software package OPAC. Bull. Amer. Meteor. Soc. (1998) 79, 831-844.

10. H. C. van de Hulst, Light Scattering by Small Particles. Wiley, New York, 1957.

11. A. Ishimaru, Wave Propagation and Scattering in Random Media. Academic Press, New York, 1978.

12. G. W. Kattawar and G. N. Plass, Radiance and polarization of multiple scattered light from haze and clouds. Appl. Optics (1968) 7, No. 8, 1519-1527.

13. P. Laven, Simulation of rainbows, coronas, and glories by use of Mie theory. Appl. Optics (2003) 42, No. 3, 436-444.

14. P. Laven, How are glories formed? Appl. Optics (2005) 44, No. 27, 5675-5683.

15. G. I. Marchuk, G. A. Mikhailov, M. A. Nazaraliev, R. A. Darbinian, B. A. Kargin, and B. S. Elepov, Monte Carlo Methods in Atmospheric Optics. Springer-Verlag, Berlin, 1989.

16. B. Mayer and C. Emde, Comment on 'Glory phenomenon informs of presence and phase state of liquid water in clouds' by A. N. Nevzorov. Atmospheric Research (2007) 84, 410-419.

17. B. Mayer, M. Schroeder, R. Preusker, and L. Shueller, Remote sensing of water cloud droplet size distribution using the backscattering glory: a case study. Atmos. Chem. Phys. (2004) 4, 1255-1263.

18. G. A. Mikhailov. Optimization of Weighted Monte Carlo Methods. Springer-Verlag, Berlin, 1992.

19. G. A. Mikhailov and M. A. Nazaraliev, The calculation of the light polarization in a spherical atmosphere by a Monte Carlo method. Izv. RAN. Fizika Atmos. Okeana (1971) 7, No. 4, 385-395 (in Russian).

20. A. N. Nevzorov, Permanence, properties and nature of liquid phase in ice-containing 
clouds. In: 11th Int. Conf. on Clouds and Precipitation, Montreal, Canada, 1992, pp. 270-273.

21. A. N. Nevzorov, Investigations in physics of liquid phase in ice-containing clouds. Meteor. Gidrol. (1993) 18, No. 9, 55-68 (in Russian).

22. A. N. Nevzorov, Cloud phase composition and phase evolution as deduced from experimental evidence and physico-chemical concepts. In: 13th Int. Conf. on Clouds and Precipitation, Reno, Nevada, USA, 2000, pp. 728-731.

23. A. N. Nevzorov, Internal mechanism of metastable liquid water crystallization and its effects on intracloud processes, Izv. RAS, Atm. Ocean Phys. (2006) 42, No. 6, 765-772.

24. A. N. Nevzorov, Some properties of metastable states of water. Physics Wave Phenom. (2006) 14, No. 1, 45-57.

25. A. N. Nevzorov, Bimorphism and properties of liquid water in cold clouds, In: Some problems of cloud physics. In: Meteorologiya i Gidrologiya, Moscow, 2008, pp. 268 298.

26. A. N. Nevzorov, Glory phenomenon and a nature of liquid-drop fraction in cold clouds. Atmos. Ocean. Optics (2007) 20, No. 8, 613-619.

27. A. N. Nevzorov, On the theory and physics of glory formation. Atmos. Ocean. Optics (2011) 24, No. 4, 344-348.

28. U. G. Oppel and G. Czerwinski, Multiple scattering LIDAR equation including polarization and change of wavelength. Proc. SPIE, Vol. 3571, pp. 14-25.

29. U. G. Oppel and H. Krasting, Retrieval of microphysical parameters from return signals of airborne and space-based LIDARs. In: Lidar Atmospheric Monitoring (Proc. European Symposium on Environmental Sensing III, 16-20 June 1997, Fairgrounds Munich, GFR. (EnviroSense'97; LASER'97) (Ed. J.-P. Wolf). Proceedings of SPIE EUROPTO Series, Vol. 3104, 1997, pp. 135-144.

30. S. M. Prigarin, The Essentials of Stochastic Simulation in Polarized Optical Radiation Transfer. Novosibirsk Univ. Press, Novosibirsk, 2010 (in Russian).

31. S. M. Prigarin, K. B. Bazarov, and U. G. Oppel, Looking for a glory in A-water clouds. Atmos. Ocean. Optics (2012) 25, No. 4, 307-313.

32. M. J. Raković, G. W. Kattawar, M. Mehrübeoğlu, B. D. Cameron, L. V. Wang, S. Rastegar, and G. L. Coté, Light backscattering polarization patterns from turbid media: theory and experiment. Appl. Optics (1999) 38, No. 15, 3399-3408.

33. J. C. Ramella-Roman, S. A. Prahl, and S. L. Jacques, Three Monte Carlo programs of polarized light transport into scattering media: Part I. Optics Express (2005) 13, No. 12, 4420-4438.

34. N. P. Romanov and S. O. Dubnichenko, Physics of formation and analytical description of glory properties. Atmos. Ocean. Optics (2010) 23, No. 7, 549-560.

35. D. Segelstein, The Complex Refractive Index of Water. M. S. Thesis, Univ. Missouri, Kansas City, 1981. 
36. E. P. Shettle and R. W. Fenn, Models for the aerosols for the lower atmosphere and the effects of humidity variations on their optical properties. AFGL-TR-79-0214 Environmental Research Papers No. 676, 1979.

37. T. A. Sushkevich, Mathematical Models of Radiation Transfer. BINOM, Moscow, 2005 (in Russian).

38. M. Theusner, Photographic observation of a natural fourth-order rainbow. Appl. Optics (2011) 50, No. 28, 129-133.

39. W. Wiscombe, Improved Mie scattering algorithms. Appl. Optics (1980) 19, No. 9, $1505-1509$. 